RAD Conference Proceedings, vol. 3, pp. 149-153, 2018

ISSN 2466-4626 (online) | DOI: 10.21175/RadProc.2018.32

www.rad-proceedings.org

\title{
IN-BEAM PET MONITORING TECHNIQUE FOR PROTON THERAPY: EXPERIMENTAL DATA AND MONTE CARLO PREDICTION
}

\author{
A. Topi ${ }^{1,2^{*}}$, G. Battistoni ${ }^{3}$, N. Belcari ${ }^{2,4}$, M. G. Bisogni ${ }^{2,4}$, N. Camarlinghi ${ }^{2,4}$, \\ A. Del Guerra ${ }^{2,4}$, A. Ferrari5, R. Kopec ${ }^{6}$, A. Kraan², D. Krzempek6 ${ }^{6}$, M. Morrocchi',4,

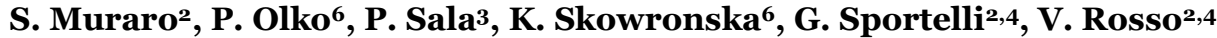

\author{
${ }^{1}$ Department of Physical Sciences, Earth and Environment, University of Siena, Siena, Italy \\ ${ }^{2}$ National Institute of Nuclear Physics (INFN), Pisa, Italy \\ 3 National Institute of Nuclear Physics (INFN), Milan, Italy \\ 4Department of Physics, University of Pisa, Pisa, Italy \\ ${ }^{5}$ CERN, Geneva, Switzerland \\ ${ }^{6}$ Institute of Nuclear Physics Polish Academy of Sciences, Krakow, Poland
}

\begin{abstract}
Charged particle therapy is a precise radiotherapy method for the treatment of solid tumors. This method can deliver conformal dose distributions minimizing damage to healthy tissues thanks to its characteristic dose profile. However, the steep dose profile of charged particle beams (due to the Bragg peak) can result in over- or under-dosage in critical regions. Monitoring the range of the charged particles is therefore highly desirable. In this study, we use a planar in-beam PET system for the range verification of pencil beams in proton therapy. The planar geometry of the DoPET system is advantageous because it can be used online, i.e., during treatment. In the particle therapy community, the Monte Carlo (MC) codes are widely used to evaluate the radiation transport and interaction with matter. For this reason, the FLUKA MC code was used to simulate the experimental conditions of irradiations performed at the Cyclotron Centre Bronowice (CCB) proton therapy center in Krakow (PL). 13oMeV pencil beams were delivered on phantoms mimicking human tissues. Different acquisitions are analyzed and compared with the $M C$ predictions. The image reconstruction for experimental data and simulation is based on the Maximum Likelihood Estimation Method (MLEM) algorithm. A special focus in the paper will be on the validation of the PET detector response for activity range verification.
\end{abstract}

Key words: Charged particle therapy, in-beam PET, FLUKA, MLEM

\section{INTRODUCTION}

Radiation therapy is an essential component of cancer therapy. The main challenge in radiotherapy for cancer treatment is how to maximize the dose to the tumor region while sparing the surrounding healthy tissue. One of the most important modalities being developed for cancer therapy is irradiation with protons and ions [1]. This modality allows the delivery of the maximum dose in well-defined volumes, significantly reducing the absorbed dose in the surroundings.

However, due to the steep dose profile of protons, this technique is much more sensitive to spatial uncertainties than conventional photon treatments. Actually, uncertainties in particle range, unexpected anatomical changes and repositioning may cause undesirable variations in dose deposition in the target or the increased dose in the surrounding tissues [2], [3]. For this reason, the creation of a system which allows the monitoring of the treatment would be desirable.

\footnotetext{
*albana.topi@pi.infn.it
}

The monitoring of particle activity distribution can be performed by detecting secondary radiation (i.e., $\beta+$ emitters, prompt gamma, and charged fragments) arising from the nuclear interaction of heavy particles with the patient tissues. Positron Emission Tomography (PET) is the most studied monitoring technique [3-6] based on the detection of back-to-back photons (511 keV) coming from e- e+ annihilation. Furthermore, since the recorded signal (i.e., the isotopic activity) and the tissue composition are directly related, the analysis of activity profiles and signal time dependence can provide additional information on the elemental composition of the irradiated object.

In this study, a dual head PET prototype jointly developed by the INFN (Instituto Nazionale di Fisica Nucleare) and the University of Pisa [6-8] is used. Irradiations were performed at the CCB proton therapy center using different phantom materials.

\section{MATERIALS AND METHODS}

The PET system used in the present research is a dual-head prototype with a planar geometry: the 
A. Topi et al., In-beam PET monitoring technique for proton therapy, RAD Conf. Proc., vol. 3, 2018, 149-153

system is named DoPET. The prototype is placed inbeam and the distance between the two heads was set at $48 \mathrm{~cm}$. Each head is composed of 9 detector modules, covering an active area of $16 \times 16 \mathrm{~cm}^{2}$. Each module is made of a $23 \times 23$ LYSO crystal matrix that is coupled to a position sensitive photomultiplier tube model $\mathrm{H} 85 \mathrm{OO}$ and read-out by custom front-end electronics. Data acquisition is performed by means of an FPGA with a time window of $3 \mathrm{~ns}$.

\subsection{Experimental setup}

The phantom and its holder are positioned in the middle of the two heads. The phantom is parallelepiped shaped with a transversal section of $5 \times 5 \mathrm{~cm}^{2}$ and a length of $14 \mathrm{~cm}$.

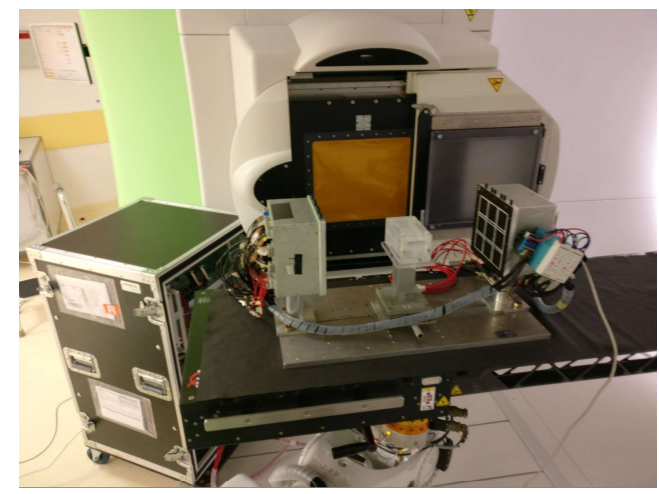

Figure 1. Picture of the experimental setup during the acquisitions at CCB Krakow. The two DoPET heads, the cables for the power supply and readout, and the flying case are visible. Behind the detecting system there is the gantry for the proton beam delivery.

The homogeneous phantoms used for the experiment are made of the following materials: polymethylmethacrylate, brain equivalent tissue, high density polyethylene and water (hereinafter referred to as PMMA, BRAIN, HDPE and WATER, respectively). In Table 1, the elemental composition and the density of each phantom are reported. The holder of the phantom is composed of PMMA and has the same length as the phantom with a transversal section of $8 \mathrm{x}$ $8 \mathrm{~cm}^{2}$.

Table 1. The list of materials of the homogeneous phantoms used. For each phantom material, the density and the elemental composition (\% by weight) are presented.

\begin{tabular}{|l|c|c|c|c|c|}
\hline Phantom & $\begin{array}{c}\text { density } \rho \\
\left(\mathrm{g} / \mathrm{cm}^{3}\right)\end{array}$ & $\mathrm{H}(\%)$ & $\mathrm{C}(\%)$ & $\mathrm{O}(\%)$ & $\mathrm{N}(\%)$ \\
\hline PMMA & 1.18 & 8.06 & 59.98 & 31.96 & \\
BRAIN & 1.05 & 10.83 & 72.54 & 14.86 & 1.69 \\
HDPE & 0.97 & 14 & 86 & & \\
WATER & 1 & 11.19 & & 88.81 & \\
\hline
\end{tabular}

The main elemental components of these phantoms are carbon and oxygen and, due to proton irradiation, the production of $\beta+$ emitters, mainly ${ }^{15} \mathrm{O}\left(\mathrm{T}_{1 / 2}=122 \mathrm{~s}\right)$ and ${ }^{11} \mathrm{C}\left(\mathrm{T}_{1 / 2}=1220 \mathrm{~s}\right)$, allows the monitoring of the delivered proton treatment. Data were acquired during irradiation (beam-on) and after irradiation (beam-off).
In this study, we will focus on the beam-off analysis considering several time intervals.

For the data collection, the DoPET prototype and the phantoms were positioned on the patient couch. A laser beam was used to verify the alignment: the experimental set-up is shown in Figure 1.

The phantoms were irradiated at the CCB proton therapy center using proton monoenergetic pencil beams (IBA proteusPLUS) with a Gaussian transversal profile $(F W H M \cong 10 \mathrm{~mm})$ and an energy of $130 \mathrm{MeV}$. Experiments were performed with high statistics $\left(10^{10}\right.$ protons) and with irradiation times (beam-on) of about $5 \mathrm{~s}$

\subsection{Data processing and image reconstruction}

Events acquired by DoPET are stored on disk in a list-mode format containing the Anger coordinates (four for each photon) and the identification code of the modules that detected the photon pair [6][9].

The Anger coordinates are used to evaluate the event barycenter and to identify the crystal where the photons interacted. Once the crystals are identified, data are calibrated into $\mathrm{keV}$. A cut in an energy window of [350, 850] keV is applied. Each pair defines a lineof-response (LOR). The resulting data are used as an input for the $3 \mathrm{D}$ reconstruction software.

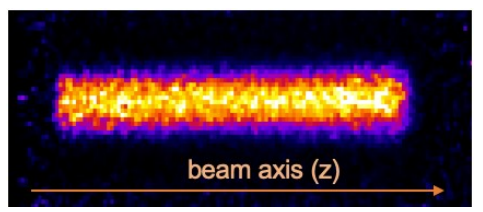

Figure 2. Reconstructed 2D image of the activated volume in the sagittal plane for PMMA phantom irradiated with 130 $\mathrm{MeV}$ pencil proton beam coming from the left.

In order to obtain the $3 \mathrm{D}$ activity image, these data first undergo a normalization procedure, based on a flat-field acquisition of a homogeneous planar phantom filled with ${ }^{18} \mathrm{FDG}$ [7], and subsequently are processed using a MLEM algorithm [10] which makes use of an analytical model of the system matrix. The reconstructed field of view (FOV) has a volume of $100 \times 160 \times 160 \mathrm{~mm}^{3}$, segmented into $1 \mathrm{~mm}^{3}$ voxels. Five iterations were performed per reconstruction. Figure 2 shows an example of the reconstructed image in the sagittal plane.

\subsection{Data analysis}

For monitoring purposes, the activity range is calculated and compared with the MC predictions. To evaluate the activity range, starting from the $3 \mathrm{D}$ activity reconstructed volume, $1 \mathrm{D}$ activity distributions are obtained projecting all the reconstructed events within a cylinder with an ellipsoidal transversal section, aligned along the beam direction (z-axis). The phantom is centered in the FOV (with $\mathrm{z}=15 \mathrm{~mm}$ ). The width of each activity profile is calculated evaluating the $50 \%$ rise and the $50 \%$ fall-off position of the $1 \mathrm{D}$ profiles fitted with Fermi-Dirac functions. The difference between the corresponding depths along the beam direction is called $\Delta \mathrm{W}_{50 \%}[11]$. 
A. Topi et al., In-beam PET monitoring technique for proton therapy, RAD Conf. Proc., vol. 3, 2018, 149-153

\subsection{FLUKA Monte Carlo simulation code}

The simulation of the experimental set-up in this study is performed with the FLUKA Monte Carlo code [12-14] reproducing the conditions of irradiations performed at the proton therapy center in Krakow.

The $\beta+$ activity and annihilation products in space and time are recorded after a detailed implementation of the detector geometry and materials. The segmented structure of the PET modules, the real geometrical LYSO crystals position, and the support framework are modelled.

We chose to use FLUKA since it has been extensively validated in particle therapy [14], its prior usage for this experimental setup [15] and because FLUKA has been thoroughly validated in studies dedicated to range monitoring using PET [5,15-16].

The expected activity profile is evaluated, and the annihilation photons are tracked from the target phantom in air and, eventually, in the detector modules. The pair of back-to-back photons reaching the two PET heads is tagged as a coincidence signal and the corresponding LOR is recorded in the data acquisition output format.

\section{RESULTS AND DISCUSSION}

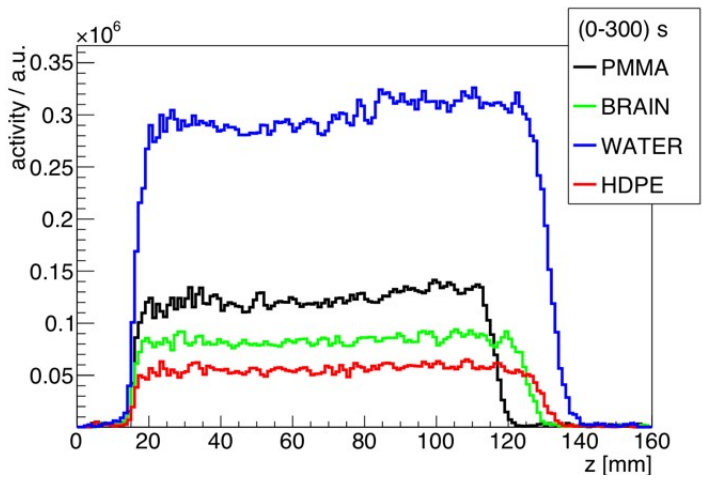

Figure 3. $1 \mathrm{D} \beta^{+}$-activity profile: (0-300) s immediately after irradiation.

Several time frames were considered to test the monitoring capabilities of DoPET. Considering a time frame of $300 \mathrm{~s}$, two different time intervals were

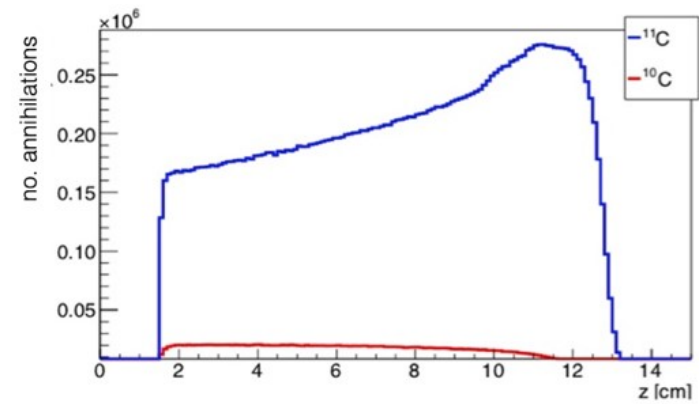

analyzed: (0-300) s and (440-740) s after irradiation (beam off). The profiles for the four homogeneous phantoms, in both time frames, are reported in Figures 3 and 4.

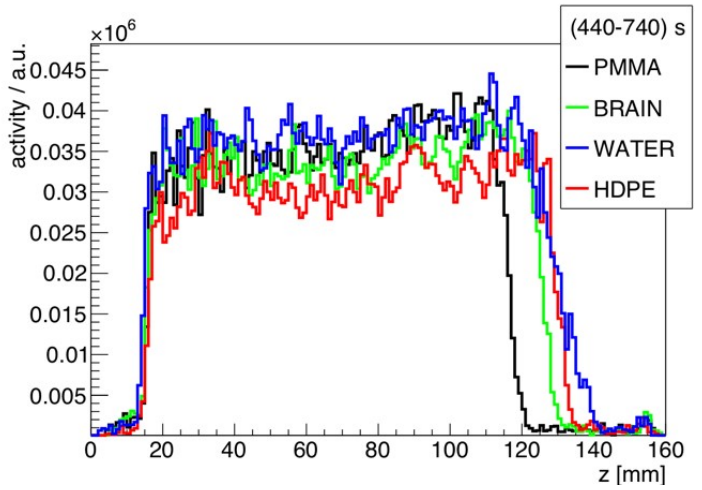

Figure 4. 1D $\beta^{+}$-activity profile: (440-740) s after irradiation.

As we can see, the total number of coincidences acquired in the delayed time frame is lower than the one collected in the acquisition that started immediately after irradiation. The different heights of the profiles are related to the elemental composition of the phantoms (see Table 1). The two major contributors to the considered phantoms are ${ }^{15} \mathrm{O}$ and ${ }^{11} \mathrm{C}$ and, by using FLUKA simulation, it is possible to calculate the $\beta+$ induced activity profiles.

The different contributions (calculated as number of annihilations vs. depth) of the two boundary cases, WATER and HDPE, are reported in Figure 5 (evaluated for 0-300 s), with only a single relevant target element $\left({ }^{15} \mathrm{O}\right.$ and ${ }^{12} \mathrm{C}$, respectively). From these isotope contribution profiles, it is possible to infer the importance of the ${ }^{15} \mathrm{O}$ signal detection. The time frame of $0-70 \mathrm{~s}$ was also considered and the relative activity profiles for the four phantoms are reported in Figure 6. Although the acquired values are lower, the four profiles are well-distinguished, additionally confirming the utility of the $15 \mathrm{O}$ signal.

Figure 5. The simulated $1 \mathrm{D}$ annihilation distribution profile in the target for a time frame of (o -300) $\mathrm{s}$ after the irradiation. Left: the profile of HDPE (contributing isotopes: ${ }^{11} \mathrm{C},{ }^{10} \mathrm{C}$ ). Right: the profile of WATER (contributing isotopes: ${ }^{15 \mathrm{O}},{ }^{11} \mathrm{C},{ }^{10} \mathrm{C}$ ). For calculation of these profiles, the tracking to reach the detector and the geometry of the detector is not taken into account. 


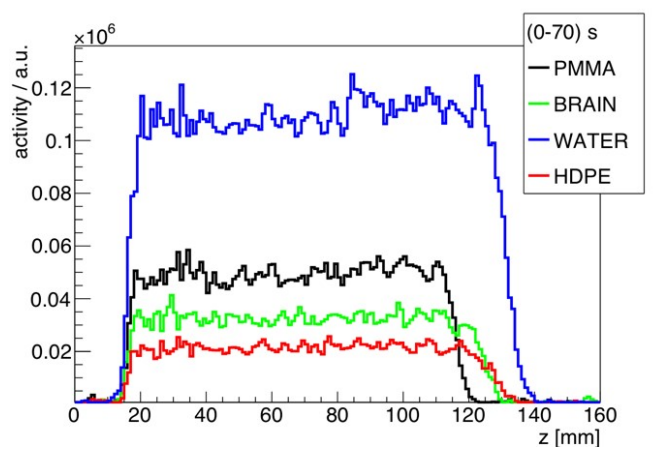

Figure 6. $1 \mathrm{D} \beta^{+}$-activity profile: (o-70) s after irradiation

It is also possible to notice that all profiles are almost flat, allowing to evaluate the mean profile height.

To demonstrate the precision of the monitoring of DoPET, the $\Delta \mathrm{W}_{50 \%}$ for the four phantoms in the time frame of (0-70) $\mathrm{s}$ was evaluated and compared to FLUKA simulations. To better see the different activity width of the four phantoms, the profiles normalized to the same unitary area are shown in Figure 7.

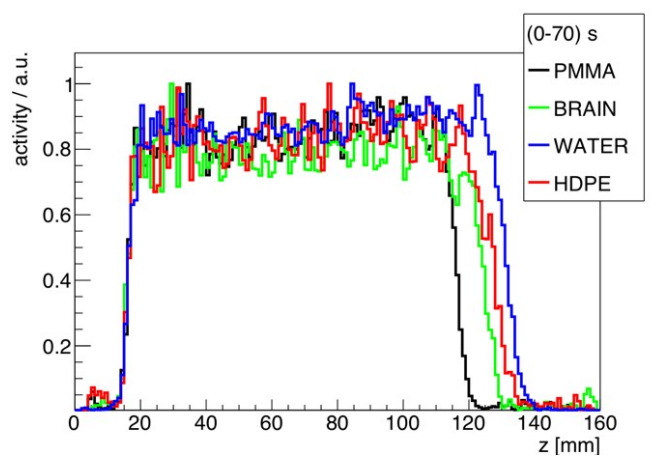

Figure 7. $1 \mathrm{D} \beta^{+}$-activity profile: $(0-70) \mathrm{s}$ immediately after irradiation. All the plots are normalized to the area.

Table 2. Activity range, $\Delta \mathrm{W}_{50} \%$, assessed for the experimental data and the corresponding FLUKA simulations (see Sec. 2.4).

\begin{tabular}{|lcc|}
\hline \multicolumn{1}{|c}{ Phantom } & $\begin{array}{c}\Delta \mathbf{W}_{\mathbf{5 0} \%} \text { exp. } \\
\text { data [mm] }\end{array}$ & $\begin{array}{c}\Delta \mathbf{W}_{\mathbf{5 0} \%} \text { FLUKA } \\
{[\mathbf{m m}]}\end{array}$ \\
\hline PMMA & $99.80 \pm 0.21$ & $100.26 \pm 0.17$ \\
BRAIN & $108.68 \pm 0.66$ & $108.08 \pm 0.38$ \\
HDPE & $112.49 \pm 0.54$ & $111.87 \pm 0.38$ \\
WATER & $114.76 \pm 0.35$ & $115.58 \pm 0.64$ \\
\hline
\end{tabular}

The evaluated $\Delta \mathrm{W}_{50 \%}$ for the four phantoms and the relative FLUKA values are reported in Table 2. It can be observed that differences between the FLUKA simulation and experimental data are within a $1 \mathrm{~mm}$ range.

\section{CONCLUSION}

In this paper, we report the tests performed with the DoPET prototype to show its monitoring capabilities. Several homogeneous phantoms were irradiated with $130 \mathrm{MeV}$ protons with high statistics pencil beams. Three different time frames were studied to point out the importance of an in-beam PET. We showed that, for an acquisition of $70 \mathrm{~s}$ after the end of the irradiation it is sufficient to distinguish the different elemental composition of the phantoms, either by the evaluated $\Delta \mathrm{W}_{50 \%}$, or by the different height of the profiles. This short time frame was studied because of the need to reduce the occupancy of the treatment room. The comparisons between experimental data and FLUKA simulations, express an agreement of the $\Delta \mathrm{W}_{50} \%$ within a mm range.

Future analyses on heterogeneous phantoms, also investigating the possibility to predict the height of the activity signal, are foreseen.

\section{REFERENCES}

1. D. Schulz-Ertner, H. Tsuji, "Particle radiation therapy using proton and heavier ion beams," J. Clin. Oncol., no. 25, no. 8, pp. 953 - 964, Mar. 2007.

DOI: $10.1200 / \mathrm{JCO} .2006 .09 .7816$

PMid: 17350944

2. A. Knopf, A. Lomax, "In vivo proton range monitoring: a review," Phys. Med. Biol., vol. 58, no. 15, pp. R131 - R160, Aug. 2013.

DOI: $10.1088 / 0031-9155 / 58 / 15 / R 131$

PMid: 23863203

3. X. Zhu, G. El Fahkri, "Proton therapy verification with PET imaging," Theranostics, no. 3, no. 10, pp. $731-740$, Sep. 2013.

DOI: $10.7150 /$ thno. 5162

PMid: 24312147

PMCid: PMC 3840408

4. A. Del Guerra et al., "Positron emission tomography: its 65 years,” Riv. Nuovo Cim., no. 39, no. 4, pp. 155 - 223, Apr. 2016.

DOI: $10.1393 / \mathrm{ncr} / \mathrm{i} 2016-10122-6$

5. K. Parodi et al., "In-beam PET measurements of $\beta+$ radioactivity induced by proton beams," Phys. Med. Biol., vol. 47, no. 1, pp. 21 - 36, Jan. 2002.

DOI: $10.1088 / 0031-9155 / 47 / 1 / 302$

PMid: 11814225

6. G. Sportelli et al., "First full-beam PET acquisitions in proton therapy with a modular dual-head dedicated system,” Phys. Med. Biol., no. 59, vol. 1, pp. 43 - 6o, Jan. 2013.

DOI: $10.1088 / 0031-9155 / 59 / 1 / 43$

PMid: 24321855

7. N. Camarlinghi et al., "An in-beam PET system for monitoring ion-beam therapy: test on phantoms using clinical $62 \mathrm{MeV}$ protons," J. Instrum., vol. 9, no. 4, Co4005, Apr. 2014. DOI: 10.1088/1748-0221/9/04/Co4005

8. L. Brombal et al., "Proton therapy treatment monitoring with in-beam PET: Investigating space and time activity distributions," Nucl. Instrum. Methods Phys. Res. A, vol. 861, pp. 71 - 76, 2017. DOI: 10.1016/j.nima.2017.05.002

9. S. Helmbrecht et al., "In-beam PET at clinical proton beams with pile-up rejection", Zeitscrift für Medicinische Physik, vol. 27, pp. 202-217, 2017

10. S. Moehrs et al., "Multi-ray-based system matrix generation for 3D PET reconstruction," Phys. Med. Biol., vol. 53, no. 23, pp. 6925 - 6945, Dec. 2008.

DOI: $10.1088 / 00031-9155 / 53 / 23 / 018$

PMid: 19001696

DOI: 10.1016/j.zemedi.2016.07.003 
A. Topi et al., In-beam PET monitoring technique for proton therapy, RAD Conf. Proc., vol. 3, 2018, 149-153

11. S. Muraro et al., "Proton therapy treatment monitoring with the DoPET system: Activity range, positron emitters evaluation and comparison with Monte Carlo predictions," J. Instrum., vol. 12, C12026, Dec. 2017.

DOI: $10.1088 / 1748-0221 / 12 / 12 / C 12026$

12. A. Ferrari, P. Sala, A. Fassò, J. Ranft, FLUKA: A MultiParticle Transport Code, Rep. CERN-2005-10, INFN/TC_05/11, SLAC-R-773, CERN, INFN, SLAC, Geneva, Switzerland, 2005.

Retrieved from:

https://www.slac.stanford.edu/pubs/slacreports/report s16/slac-r-773.pdf;

Retrieved on: May 12, 2018

13. T. T. Boehlen et al., "The FLUKA code: developments and challenges for high energy and medical applications," Nucl. Data Sheets, vol. 120, pp. 211 - 214, Jun. 2014.

DOI: $10.1016 /$ j.nds.2014.07.049
14. G. Battistoni et al., "The FLUKA code: an accurate simulation Tool for Particle Therapy," Front. Oncol., vol. 6, no. 116, May 2016.

DOI: $10.3389 /$ fonc.2016.00116

PMid: 27242956

PMCid: PMC4863153

15. V. Rosso et al., "A new PET prototype for proton therapy: Comparison of data and Monte Carlo simulation,”J. Intrsum., vol. 8, Co3021, Mar. 2013. DOI: $10.1088 / 1748-0221 / 8 / 03 / C 03021$

16. A. Kraan et al., "Online monitoring for proton therapy: A real-time procedure using a planar PET system," Nucl. Instrum. Methods Phys. Res. A, vol. 786, pp. 120 - 126, Jun. 2015.

DOI: 10.1016/j.nima. 2015.03.059 